\title{
Case Report of a rare occurrence of multiple metachronous malignant neoplasm in a female patient
}

\author{
Sakthiraj $\mathrm{N}^{1}$, Getheeswaran $\mathrm{S}^{2}$, Uthayakumaran $\mathrm{S}^{2}$
}

\section{Summary}

It is a rare case of a 53 year old female patient with three different malignancies occurring metachronously; papillary carcinoma of the thyroid followed by malignant phyllodes tumor of her left breast and finally acute myeloid leukaemia. As the patient in the report had received postoperative local ionising radiation treatment seven years prior to her development of acute myeloid leukaemia, the role of the exposure to ionising radiation as the causative mechanism in the development of this malignancy cannot be completely ruled out. This case report suggests that patients who have had a first cancer successfully treated are at higher risk of developing a second or even a third subsequent cancer; therefore, long term regular followup is essential for successfully treated cancer patients for the early diagnosis of the development of subsequent malignancies and their effective treatment.

\section{Background}

Although the occurrence of multiple synchronous or metachronous malignant neoplasia in patients remains very rare, the improving early diagnosis and effective treatment of many neoplasia could result in more frequent occurrence of multiple neoplasia in future.

This case report describes a rare case with three different neoplasia occurring at different times, within a period of seven years; two of which were successfully treated. It is therefore important to carryout regular follow up of successfully treated patients for malignancies in order for the early detection of subsequent malignancies to provide effective treatment.

\section{Case presentation}

A 53 year old married female patient, with no children, employed as a school principal, was transferred from the local hospital for further investigations as she had persistent pancytopenia, fever and lethargy for the previous 10 days. She was a non-smoker with no family history of malignancies.
According to her past medical history, she had developed papillary carcinoma of the thyroid gland before seven years which was localised with no evidence of spread and was treated with total thyroidectomy followed by local ionising radiation therapy and replacement thyroxin therapy. She remained well following the treatment; but after four years, she again presented with a malignant phyllodes tumor in her left breast that was treated by wide local excision and histology confirmed clear excision margins. However, after a year she developed a small local recurrence of the tumour which was then completely excised and confirmed by biopsy. Then, the patient remained well for the following three years. Later, she became unwell and a bone biopsy together with flowcytometry confirmed that she had developed acute myeloid leukaemia to which she succumbed three weeks later.

\section{Discussion}

Multiple malignant tumors have become an accepted and recognized phenomenon since it was first described in 1878, a case of splenic leukaemia and carcinoma of the pancreas by Whipham followed by Billroth reporting multiple primary cancers of the breast in 1879(1). Multiple primary malignancies are defined as those that are confirmed, independent, and of non-metastatic origin and they are classified as synchronous if they are identified within the first six months after the appearance of the first lesion or as metachronous if they are identified thereafter(2). The overall prevalence of multiple primary malignancies is reported as $0.7-11.7 \%$, increasing proportionally with patient's age $(2,3,4)$. It is estimated that $75 \%$ of cases occur in individuals over 50 years of age 2 . These values are on the rise due to the increased longevity of the population and improved lifestyles, the improvement of diagnostic methods and the variety and effectiveness of treatments now available $(2,4)$. Hayat et al.(3) reported a probability of developing a second malignancy, depending on the primary tumors diagnosed, ranging from 1\% (history of hepatic neoplasia) to $16 \%$ (previous bladder tumors)3. Braisch et al.(5) noted that $1.2-2.5 \%$ of cancer patients 
who were smokers developed another malignant lesion within the first year of follow-up.

The second malignant neoplasm that developed in our patient, the malignant phyllodes tumor, is a very rare breast tumor with a reported incidence of 0.3-0.9 (6) and there are yet no reports of it occurring as a subsequent tumor in a patient.

According to the classification of the World Health Organization, therapy-related myeloid neoplasms can be divided into two subtypes: (1) an alkylating agent/ radiotherapy-related type; and (2) a topoisomerase II inhibitor-related type(7). The development of therapyrelated myelodysplastic syndromes (t-MDS) and therapyrelated acute myeloid leukemia ( $\mathrm{t}$-AML) after alkylating agents or radiotherapy usually occur after a median latency of 4-7 years, with two-thirds of patients presenting with MDS and one-third presenting with $\operatorname{AML}(8,9)$.

Although only a small section of the bone marrow of the patient in the case report was exposed to ionising radiation, seven years previously following thyroidectomy for Papillary Carcinoma, the development of therapy induced Acute Myeloid leukaemia cannot be excluded.

This case report and review suggests that patients who have had a first cancer and treated successfully are at higher risk of developing a subsequent cancer; and regular follow-up with thorough examination of such patients could lead to the early detection and effective treatment of a subsequent cancer.

\section{Learning points/Take home messages}

- Multiple malignant neoplasms are rare but increasing in incidence and can occur synchronously or metachronously in previously treated cancer patients.

- The probability of developing a second malignancy is higher in patients over 50 years of age, previously treated for bladder cancer and smokers.

- Although rare, the development of therapy related Acute Myeloid Leukaemia needs to be kept under consideration.

\section{References}

1. Cornes JS.Multiple primary cancers: primary malignant lymphomas and carcinomas of the intestinal tract in the same patient. J. clin. Path. (1960), 13, 483.Available from: https://www.ncbi.nlm.nih.gov/pmc/articles/ PMC480126/pdf/jclinpath00059-0027.pdf

2. Demandante CG, Troyer DA, Miles TP, et al. Multiple primary malignant neoplasms case report and a comprehensive review of the literature. Am J ClinOncol. 2003;26:79-83.Available from: https://www.ncbi.nlm. nih.gov/pubmed/12576929

3. Hayat MJ, Howlader N, Reichman ME, et al. Cancer statistics, trends, and multiple primary cancer analyses from the Surveillance, Epidemiology, and End Results (SEER) Program. The Oncologist. 2007;12:20-37. doi:10.1634/theoncologist.12-1-20

4. VanderWalde AM, Hurria A, et al. Second malignancies among elderly survivors of cancer. The Oncologist. 2011;16:1572-1581.doi: doi:10.1634/ theoncologist.2011-0214

5. Braisch U, Meyer M, Radespiel-Tröger M, et al. Risk of tobacco-related multiple primary cancers in Bavaria, Germany. BMC Cancer. 2012;12:250-250. Available from: https://doi.org/10.1186/1471-2407-12-250

6. Bernstein L., Deapen D. The descriptive epidemiology of malignant cystosarcomaphyllodestumors of the breast. Cancer. 1993;71:3020-3024. doi: 10.1002/1097-0142 (19930515)71:10<3020::AIDCNCR2820711022>3.0.CO;2-G

7. Vardiman JW, Harris NL, Brunning RD. The World Health Organization (WHO) classification of the myeloid neoplasms. Blood. 2002;100:2292-2302. DOI:10.1182/ blood-2002-04-1199

8. Bhatia S. Therapy-related myelodysplasia and acute myeloid leukemia. SeminOncol. 2013;40:666-675. doi: 10.1053/j.seminoncol.2013.09.013

9. Karp JE, Sarkodee-Adoo CB. Therapy-related acute leukemia. Clinics in Laboratory Medicine. 2000;20:71-81, ix. Available from: https://www.ncbi.nlm.nih.gov/ pubmed/10702897 